\title{
Demiarcs, Creaons and Genons
}

\author{
ROBERT W. BOSSERMAN \\ Systems Science Institute, University of Louisville, Louisville, \\ Kentucky 40292
}

AND

Frank Harary

Research Center for Group Dynamics, Institute for Social Research, University of Michigan, Ann Arbor, Michigan 48106, U.S.A.

(Received 11 August 1980, and in revised form 9 March 1981)

Useful insights into the representation of natural systems can be gained by decomposing directed graphs (digraphs) into elementary components. Arcs of digraphs can be split into male demiarcs (outarcs) which leave vertices and female demiarcs (inarcs) which enter demiarcs. Likewise, a vertex can be split into an input perceiving side called the creaon and an output generating side called the genon. Digraphs can be regarded as being hierarchically organized because each vertex in a level-1 digraph can be expanded into a level-2 digraph. In general, each vertex of a level- $i$ digraph can be expanded into a level- $(i+1)$ digraph. Arcs of a level- $i$ digraph can be regarded as bundles of level- $(i+1)$ arcs which are split at the vertex boundary. These elementary graphical components are shown to be useful for depicting input-output systems such as organisms, ecosystems and societies.

\section{Introduction}

In the development of fields of mathematics and science, it is often useful to reduce problems to elementary parts. From these elementary parts and their interrelations, new perspectives and understandings are gained. Recently the decomposition of graphs into elementary or atomistic parts has been considered (Harary, 1971; Harary \& Havelock, 1972). Related work has been done with the decomposition of abstract objects into elementary parts (Patten et al., 1976). By combining the results from the graph theory and systems theory, more complete understanding of the relationships between graphs and system representations can be accomplished. 


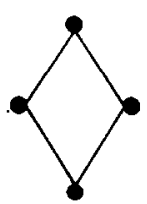

(a)

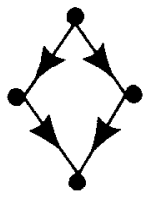

(b)

Fig. 1. (a) A graph and (b) a digraph.

\section{Elementary Components of Graphs}

A graph is a set of points together with a set of unoriented lines each of which joins two of these points. The points are usually called vertices and the lines are edges (Harary, 1969). Generally, edges joining a vertex to itself (loops) are not allowed in a graph. A directed graph or digraph is composed of vertices and oriented lines called arcs. Examples of a graph, and a digraph are shown in Fig. 1. An edge in a graph (Fig. 2a) can be regarded as being the composite of two oppositely oriented arcs (Fig. 2b).

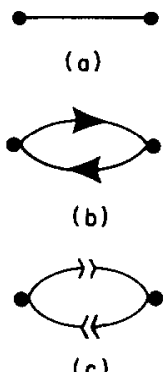

(c)

FIG. 2. Decomposition of (a) an edge into (b) two oppositely oriented arcs, and further into (c) male and female demiarcs.

Each arc can be further decomposed into a male and female demiarc (Fig. $2 c$ ). The male demiarc (Fig. 3a) goes from a vertex, whereas the female demiarc (Fig. 3b) goes to a vertex (Harary, 1971)†. These demiarcs join, or mesh at a junction (Fig. 3c). Male demiarcs must mesh with a compatible female demiarc to form an arc. Demiarcs which are meshed are said to bound and those which are not meshed are said to be free. Associated with

† Note: The designation male and female is consistent with general biological and mechanical definitions, and is not meant to have societal connotations. The following statement establishes an appropriate relationship between male and female demiarcs: "a part ... fitting into a corresponding female part" (The Random House Dictionary of the English Language, 1968). Terms such as inarc and outarc could also be used in place of female and male demiarcs. 


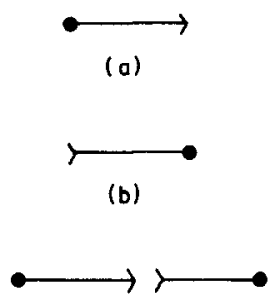

(c)

FIG. 3. (a) A male demiarc, (b) a female demiarc, and (c) an arc comprised of two bound demiarcs.

a vertex may be any number of free or bound demiarcs. The processes by which demiarcs become compatible and mesh to form arcs and digraphs have implications in a number of fields such as chemistry, sociology and electrical engineering (Harary, 1971). The decomposition of edges suggests that vertices can be similarly decomposed into elementary parts. Such a vertex decomposition has been used in systems ecology to analyze and understand the structure of ecosystems (Patten et al., 1976; Patten, 1975, 1978; Patten \& Auble, 1980). This decomposition will be discussed in the next section.

\section{Elementary Components of Systems}

In mathematical systems theory, a system can be defined as an interconnected set of abstract objects (Zadeh \& Desoer, 1963). An abstract object, $A$, can be defined by a set of attributes which is assigned to the object, e.g. $A=\left\{a_{0}, a_{1}, a_{2}, \ldots\right\}$. If the abstract object represents a real object or phenomenon, these attributes can be observables which are established by some measuring instrument or meter (Rosen, 1978). If the abstract object represents a mathematical object, the attributes can be numbers, subsets, or functions. Abstract objects interact through attributes which are called terminal variables. Initially the attributes of an abstract object can be regarded as being unoriented (Fig. 4a), however they can be oriented to form the input, output, and state subsets of the object (Fig. 4b). Inputs, which are oriented towards the abstract object, can be regarded as causes that influence the behavior of the object. Outputs, which are oriented away from the abstract object, can be regarded as characteristics of the abstract object which are useful in relating inputs to outputs. Different abstract objects are coupled through their input-output sets such that the outputs of one object become the inputs to the other. 


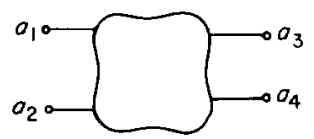

(a)

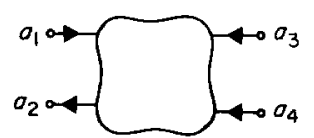

(b)

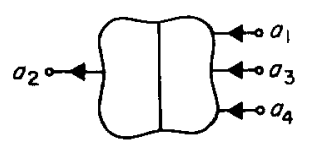

(c)

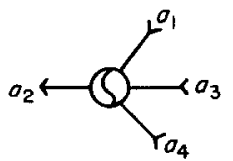

(d)

FIG. 4. Symbolic representation of (a) an abstract object with unoriented attributes, (b) an abstract object with oriented attributes, (c) an abstract object decomposed into the input side (genon) and output side (creaon), and (d) a graphical representation of the elementary parts of an abstract object.

One of the goals of systems identification, modelling and analysis is to relate the inputs of an abstract object to the outputs by some mapping or transfer function (Zadeh \& Desoer, 1963; Miller, 1978; Mesarovic \& Takahara, 1975). This mapping may be simple or complex depending on the behavior of the abstract object. State variables are often introduced to make into a function the mapping between inputs and outputs. The inputoutput mapping for a passive object such as a rock can be simple, whereas the input-output mapping for a living system can be complex. For example, the relationships between the inputs and outputs of an organism are mediated by a complicated information processing system (Von Bertalanffy, 1962).

The inputs and outputs of an abstract object and the resulting systems can easily be modelled by a digraph. If the abstract object is represented by a vertex, then the inputs and outputs of the abstract object can bc represented by the female and male demiarcs of the vertex, respectively (Fig. 4d). 
An abstract object can be partitioned into elementary parts (Fig. 4c) which correspond to the input side and the output side of an abstract object (Patten, 1975; Patten et al., 1976). The input side has been called the creaon because of its "selective role in defining a perceived environment" (Patten et al., 1976). The creaon perceives and selects attributes from the environment, whereas the genon generates and directs outputs to the environment. The relationship between the genon and creaon can be represented by a mapping or transfer function between inputs and outputs. Although this partitioning of an abstract object into dual components is consistent with the behavior of simple objects, it is most appropriate for living and man-made systems. For example, the input-output relations of a rock require no mechanisms for perception, selection, creation, and direction of matter, energy and information flows. In living systems, inputs and outputs are often partitioned into discrete structures which embody perception, input selection, output generation and direction (Miller, 1978). Advanced organisms, for example, have distinct organs or gates (Platt, 1969) for dealing with inputs and outputs of matter, energy and information. Inputs to a living system are often encoded to the internal language of the system, necessitating non-trivial mappings for the representation of the relations between inputs and outputs. Likewise, social systems such as cities have distinct input and output gates which translate matter, energy, and information flows to and from the internal language of the system (Platt, 1969; Miller, 1978).

The distinction between the genon and creaon of an abstract object can be represented in graph theory by regarding vertices as being split into an input side and output side. Female demiarcs enter the creaon side of the vertex, whereas male demiarcs leave the genon side of the vertex. The creaon and genon might be thought of as the feminine Yin and the masculine Yang of Chinese philosophy (Fig. 5) in order to represent the mutual dependence of the two sides of a vertex or abstract object. Like the Yin and the Yang, the interaction between the genon and creaon "influences the destinies of the creatures and things" (The Random House Dictionary of the English Language, 1968).

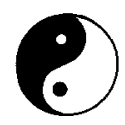

(a)

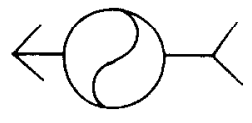

(b)

Fig. 5. (a) The Yin and Yang of Chinese philosophy. The elementary parts of a graph: male demiarc (outarc), female demiarc (inarc), genon and creaon. 
The nested-digraph representation of a hierarchical system (Harary \& Batelle, 1981) can be analyzed fruitfully in terms of an elementary component. Consider a digraph that models a hierarchical system and is composed of several vertices and arcs. Such a digraph has been called the level-1 digraph of the system (Harary \& Batelle, 1981). Each vertex of the level-1 digraph can be expanded to a level-2 digraph to represent the detailed structure of a subsystem. Vertices of the level-2 digraph can be further expanded into level-3 digraphs; in general, vertices of a level- $i$ digraph can be expanded into level- $(i+1)$ digraphs. In Fig. 6 a vertex of
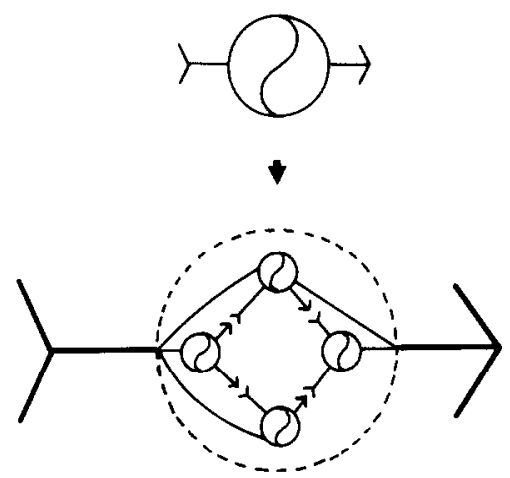

FIG. 6. The expansion of a level-1 vertex and its demiares into a level-2 digraph.

a level-1 digraph and its demiarcs are expanded into a level-2 digraph. Each demiarc in the level-1 digraph can be considered to a bundle of demiarcs which interconnect the level-2 digraphs. In Fig. 6 these bundles of demiarcs are split into individual demiarcs at the boundary of the subsystem. The level-2 digraph of an expanded vertex can denote a mapping between the inputs and outputs of the expanded vertex. Vertices can be increasingly disaggregated in order to make more precise representations of the mapping. The most useful decomposition of a vertex would be into vertices which represent "elementary natural subsystems" onto which distinct modes of behavior can be isolated (Rosen, 1978; Mesarovic \& Takahara, 1975). Such hierarchical representations should be useful for the analyses and interpretation of complex biological, social, ecological, and economic systems.

\section{Implications for Natural System Representation}

Free demiarcs can be used to represent the points where a real object interfaces with its environment. A real object always interacts with other 
objects in the environment, so that whenever the object is conceptualized or modelled, many of these interactions are abstracted away (Bunge, 1959). The actual dissection or isolation of a real object from its environment removes many of the natural couplings between the object and other objects. This process can be represented as creating free demiarcs from naturally bound demiarcs. As new objects are added to the conceptualization or model, formerly free demiarcs are bound to the new object.

The existence of a free demiarc in a graph is probably foreign to most graph theorists, because being bound within a digraph is the demiarc's natural (static) condition. A free demiarc almost demands to have itself bound to another and therefore reflects the natural propensity of interaction that occurs in many natural systems. Often a great deal of energy must be expended to keep potential interactions from occurring. For example, the exploitation of a crop by a herbivore might be regarded as an arc oriented in the direction of energy and matter flow; that is, from the crop to the herbivore (Fig. 7). The demiarc from crops represents the resources which can potentially be exploited. The demiarc into herbivores represents the capability and needs of the herbivore to exploit the crop. Energy and pesticides must be applied to keep this potential interaction from happening, i.e. to keep the two demiarcs from becoming bound (Fig. 7b).

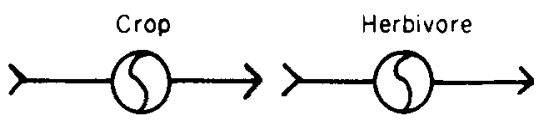

(a)

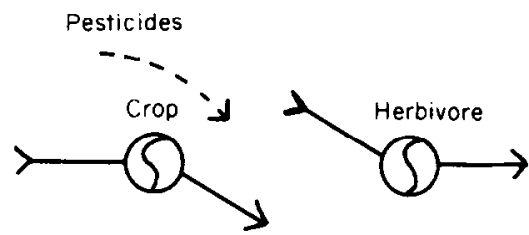

(b)

Fig. 7. A crop-herbivore interaction. (b) The application of pesticides which breaks the crop-herbivore interaction.

In many disciplines there are principles which indicate that when interactions between sympatric objects are possible they will eventually occur (Harary \& Havelock, 1972). In chemistry, for example, when two molecules are confined to the same container and have the potential to react, they generally will react. The existence of free electrons which can be bound 
in a chemical reaction are represented by free demiarcs that are oriented according to their relative electronegativities.

In biochemistry, the reaction of molecules is often mediated by an enzyme. One can depict an enzyme, its substrate, its coenzyme, and its product by vertices with free demiarcs that can become bound during the enzymatic reaction (Fig. 8). Bound arcs represent the uptake and release of molecules. Free demiarcs in Fig. 8 depict the interactions of the enzyme system with the environment, e.g. the free male demiarc from the product represents utilization by reactions in the environment. The selectivity of the enzyme is represented by the creaon/genon dichotomy.

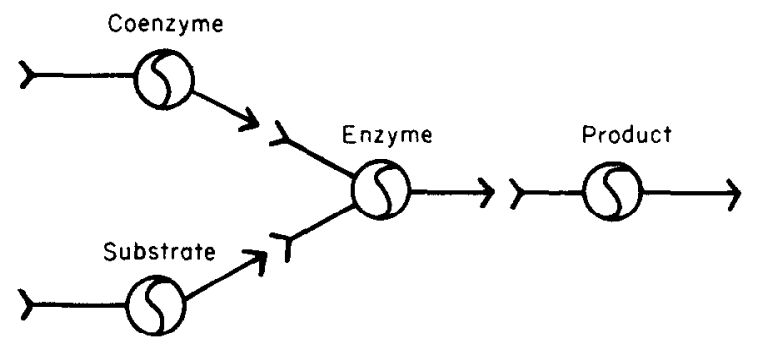

FIG. 8. An enzyme, its substrate, its coenzyme, and the product of the enzymatic reaction.

In ecology, an analogous principle about the inevitability of interaction is embodied in the principle that an available niche will eventually be filled by some species (Dice, 1952). The interaction between a species and its environmental resources constitutes the species' niche. When a niche is unfilled in an ecosystem there are resources which are not utilized (Fig. 9a). Free demiarcs in Fig. 8 denote interactions with the ecosystem environment. An available niche can be represented as a set of free demiarcs which can be found by the free demiarcs of a compatible species (Fig. 9b). Available niches can be formed either by the expansion of the resource base or the removal of another species. Niches can be filled by a new species or by increased resource utilization by a species already present. The filling of a niche is not passive but involves active selection of inputs and active creation and direction of outputs by the organisms involved. The coupling between genon and creaon, which represents the relationship between inputs and outputs of energy and matter resources, is essential to the niche filling process. In an integrated ecosystem, not only must the inputs of a species be compatible with the other species, but the outputs, which represent inputs to other species, must also be compatible. Introduction of a new species will probably result in the reshuffling of resources in the ecosystem. This phenomenon can be represented by the breaking and 


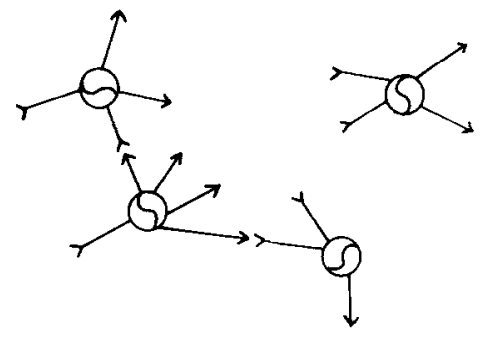

(a)

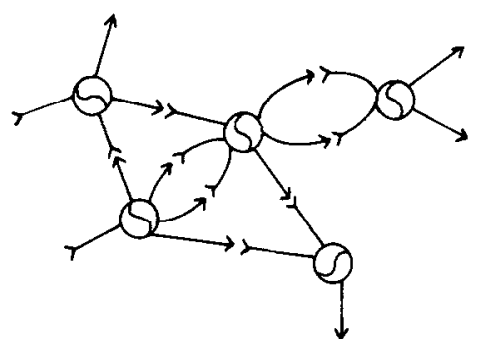

(b)

FIG. 9. (a) An unfilled niche with free demiarcs as interactions with the environment. (b) A niche which has been filled with a compatible species.

rejoining of demiarcs, and as being directed by the interrelations between genons and creaons of species vertices. The ecosystem configuration that results from the active interaction of species is different from that of a system that would result from the passive interaction of species.

\section{Graphical Representation of Utricularia-periphyton Ecosystems}

An application of these concepts to Utricularia-periphyton ecosystems in Okefenokee Swamp is shown in Fig. 10. Utricularia is a carnivorous, aquatic plant which floats freely in the water column of freshwater marshes such as in Okefenokee Swamp. Associated with Utricularia are aquatic organisms which are attracted to the organic materials secreted by Utricularia and by the favorable microenvironment produced by this netlike plant (Edmondson, 1944, 1945; Wallace, 1978; Bosserman, 1979). Utricularia often has the greatest diversity of periphyton of any sympatric macrophyte. All classic ecosystem processes (e.g. primary production, herbivory, carnivory, decomposition, nitrogen fixation and nutrient cycling) have been identified in these microcosms (Bosserman, 1979). The near 


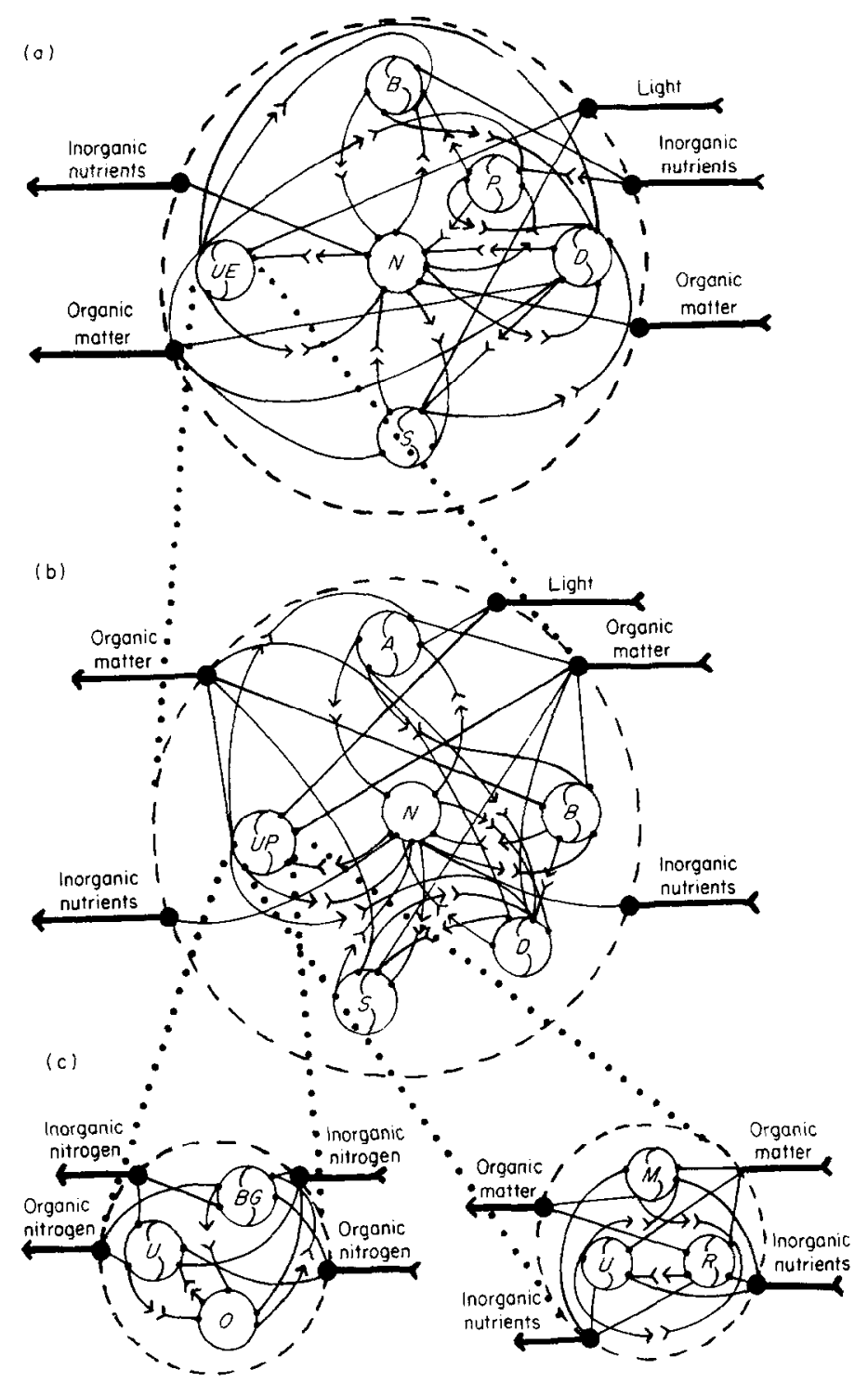

FIG. 10. Digraph of Utricularia-periphyton ecosystems in marshes of Okefenokee Swamp. (a) Relationships of ecosystems to their marsh environment. (b) Major components of ecosystem. (c) Specific relationships between Utricularia and certain tightly coupled components of periphyton. $U E=$ Utricularia-periphyton ecosystems, $B=$ biophages, $S=$ saphrophages, $D=$ detritus, $\mathrm{P}=$ aquatic plants, $N=$ inorganic nutrients, $A=$ algae, $U P=$ Utricularia and periphyton, $B G=$ bluegreen algae, $\mathrm{O}=$ organic matter, $m=$ mucus-bacteria complex, $R=$ rotifer, and $U=$ Utricularia. 
balance between production and respiration as indicated by the $P / R$ ratio indicates that the ecosystems are metabolically integrated wholes which are near their climax states. In order to achieve this integration on a microscale, organisms orient themselves in order to optimize their inputs and outputs of matter and energy. The macroscopic expression of this dynamic optimization is a stable configuration of energy and matter flows which can be acted on by external organisms. Such stable and complex configurations are only possible in a self-organizing system which is far from thermodynamic equilibrium (Jantsch, 1980). Because the structure and function of a Utricularia-periphyton ecosystem can best be described on several levels of organization (Odum, 1971), the ability to disaggregate digraphs into digraphs of different levels is useful and appropriate.

In Fig. 10 is a level-1 digraph in which the Utricularia-periphyton ecosystems $U E$ are shown as part of the more inclusive marsh environment of Okefenokee Swamp. Each vertex with associated demiarcs can be disaggregated into a more detailed digraph which indicates the input-output mappings giving rise to holistic behavior in the suprasystem. Inputs of light, inorganic nutrients, and organic matter drive the marsh ecosystem away from thermodynamic equilibrium, while gradients of energy and chemicals in the water column constrain the distribution of organisms and ecosystem processes. Many marsh organisms actively move to find an optimal environment which in an integrated ecosystem is altered by the presence of other organisms; often the outputs of one organism become the inputs to another organism. This active orientation is expressed by the dichotomy between genon and creaon and the meshing of compatible demiarcs. Inorganic nutrients, of course, are incapable of such active selection and movement; therefore, they are not depicted as having a genon and creaon, although they are selected by organisms. This distinction between non-living and living objects is compatible with the observation of Patten \& Auble (1980) that organisms make many-to-one mappings from inputs to outputs while non-living objects do not. Biophages $B$ and saprophages $S$ in the environment shape the external characteristics and composition of the Utriculariaperiphyton ecosystems. The result of ecosystem interactions with external consumers and environmental gradients is the formation of a distinct boundary composed of relatively inedible and light-tolerant species (Bosserman, 1979). Aquatic plants $P$, such as Nymphaea (white water lily), Nuphar (yellow water lily), and Orontium (golden club), also play important roles in the marsh ecosystems, providing food and detritus $D$ for other organisms and altering the dissolved nutrient concentrations.

The vertex $U E$ which represents Utricularia-periphyton ecosystems in Fig. 10a is expanded in Fig. 10b into a level-2 digraph in order to 
demonstrate the vertices and demiarcs which compose it. There are, of course, many ways to disaggregate such an ecosystem. In Fig. $10 \mathrm{~b}$ the ecosystem has been disaggregated according to the strategy of Wiegert \& Owen (1971) so that it contains both a grazing and detrital food chain. The UP vertex represents Utricularia and the periphyton which is most tightly coupled to the Utricularia plant. Periphyton components include many types of desmids, a bluegreen alga, diatoms, rotifiers, and bacteria (Bosserman, 1979). Also in the ecosystem are many algae $A$ which are not closely associated with Utricularia. These algae include those found in shallow, stagnant waters such as the light-tolerant Zygnema, Spirogyra, and Muegeotia, which form a thick layer at the top of the ecosystems. The biophages $B$ and the saprophages $S$ include a diverse set of rotifiers, cladocerans, aquatic insect larvae, oligochaetes, and mites. The detritus vertex $D$ includes the feces, carcasses, plant fragments, dissolved organic matter, mucus, bacteria and fungi present in the ecosystem. This collection of material is the biodetritus of Odum \& de la Cruz (1963) and is the major site of decomposition and nutrient mineralization. Detrital food chains dominate the Utricularia-periphyton ecosystems, even though detritus processing is inhibited in the marsh as a whole.

Two alternative schemes of expanding the vertex $U P$ of Fig. 10b are shown in Fig. 10c. In the first scheme, the relationships between Utricularia and the bluegreen alga Haplosiphon are examined. Haplosiphon, which is sometimes dominant in the Okefenokee algal community (Frohne, 1942) fixes nitrogen at rapid rates (Bosserman, 1979), thus providing a nitrogen source for organisms in the Okefenokee marshes. Haplosiphon is a branched bluegreen that is intertwined among the Utricularia plants and other algae; its existence in the acidic $(\mathrm{pH}=4.0)$ water of Okefenokee Swamp is contrary to most bluegreen algae which cannot survive in waters that have $\mathrm{pH}$ less than 4.0 (Shapiro, 1973). Utricularia and its associated periphyton obtain nitrogen from Haplosiphon; while, in exchange, Haplosiphon receives organic matter $O$ from the plants and is able to exist in the more favorable microenvironment provided by the Utricularia-periphyton ecosystem. Nitrogen fixation is an energy intensive process which often requires energy subsidies from autotrophs in order to occur (Owen \& Wiegert, 1976). Utricularia has many sugar and mucus secreting glands on its epidermis, which according to some observers secrete copious amounts of carbohydrates (Lloyd, 1933; Wallace, 1978). Many of the desmids and other green algae also secrete organic materials which can be used by Haplosiphon. The mutualistic relationships between the bluegreen alga, Utricularia and green algae are probably necessary to maintain productivity in this nitrogen deficient but energy rich environment (Bosserman, 1979). 
In the second scheme a commensal relationship between $U$ tricularia $U$ and the rotifer Ptygura beauchampii $R$ is depicted. The relationship is mediated by a mucus bacteria complex $M$ which is formed from mucus that is secreted by glands in the trichomes of Utricularia bladders and is then colonized by bacteria (Wallace, 1978). Larval Ptygura are attracted to the bladders by organic compounds secreted by glands on the Utricularia plants. The rotifer attaches itself to the trichomes on the bladder and thereby takes advantage of the quiescent microclimate provided by the Utricularia ecosystems (Edmondson, 1944, 1945; Bosserman, 1979; Wallace, 1978) and the energy and nutrients provided by Utricularia and its periphyton. This relationship is so specific that Ptygura can only be found on the bladder hairs of Utricularia (Wallace, 1978).

\section{Conclusion}

The synergistic relationship which has occurred in the past between graph theory and system theory is well known. Graphical interpretations of systems have generated many non-trivial perspectives and analyses. Recently, for example, hierarchical systems have been defined in terms of sets of nested graphs (Harary \& Batell, 1981). Results from either discipline can be interchanged profitably so that new methods and techniques emerge. The decomposition of graphs and systems into atomistic or elementary parts, as done in this paper, should have positive effects in both graph theory and system theory.

In summary, the splitting of edges into arcs, and further into demiarcs, denotes the dynamic interactions that occur between two systems. Many systems have variable structure with interactions that are constantly broken and remade. Therefore, interactions which exist at one point in time may not exist at another. Decomposition of arcs into demiarcs depicts the potential variability of system structure which is important in any selforganizing object. The junction between a male and female demiarc is analogous to the channel that separates two communicating systems (Shannon \& Weaver, 1949). The splitting of vertices into creaons and genons is a representation of the selective and generative abilities of objects. Such a decomposition of graphical components should be most valid and useful for describing man-made and living systems with complicated communication patterns and non-trivial transfer functions that map inputs into outputs (Miller, 1978; Patten \& Auble, 1980). An elaboration of the niche concept in ecology has been gained by examining abstract objects in terms of elementary components (Patten \& Auble, 1980). A hierarchical representation of a diverse ecosystem has demonstrated the utility in dcpicting 
ecological phenomena at different levels of organization. Similarly, the decomposition of graphs into demiarcs, genons, creaons, and higher level graphs should provide new perspectives and representations for other hierarchically organized systems in social, psychological and economic disciplines.

Dr B. C. Patten reviewed the manuscript and provided valuable criticism. We would like to dedicate this paper to James Grier Miller on his 65 th birthday.

\section{REFERENCES}

Bosserman, R. W. (1979). The Hierarchical Integrity of Utricularia-Periphyton Microecosystems. Ph.D. Dissertation. University of Georgia, Athens, Georgia.

Bunge, M. (1959). Causality. Cambridge, Massachusetts: Harvard University Press.

DiCE, L. R. (1952). Natural Communities. Ann Arbor: University of Michigan Press.

EDMONDSON, W. T. (1944). Ecol. Mono. 14, 31.

EDMONDSON, W. T. (1945). Ecol. Mono. 15, 141.

Frohne, W. C. (1942). U.S. Public Health 54, 1209.

HARARY, F. (1969). Graph Theory. Reading, Massachusetts: Addison-Wesley.

HARARY, F. (1971). J. Math. Soc. 1, 195.

HARARY, F. \& BATELL, M. F. (1981). Social Networks, to appear.

HARARY, F. \& HAVELOCK, R. G. (1972). Human Relations 25, 413.

JANTSCH, E. (1980). The Self-Organizing Universe: Scientific and Human Implications of the Emerging Paradigm of Evolution.

LLOYD, F. E. (1933). Can. J. Res. 13, 234.

Mesarovic, M. D. \& TAKAhARA, U. (1975). General Systems Theory: Mathematical Foundations. New York: Academic Press.

MILlER, J. G. (1978). Living Systems. New York: McGraw-Hill.

ODUM, E. P. (1971). Fundamentals of Ecology. Philadelphia, Pennsylvania: W. B. Saunders,

Odum, E. P. \& DE LA CRUZ, A. A. (1963). AIBS Bulletin 13, 39.

OWEN, D. F. \& WIEGERT, R. G. (1976). Oikos 27, 488.

PAtTen, B. C. (1975). In: New Directions in the Analysis of Ecological Systems (G. S. Innis, ed.). p. 1. La Jolla, California: Society for Computer Simulation.

PatTen, B. C. (1978). Ohio J. Sci. 78, 206.

Patten, B. C. \& Auble, G. T. (1980). Synthese 43, 155.

Patten, B. C., Bosserman, R. W., Finn, J. T. \& Cale, W. G. (1976). In: Systems Analysis and Simulation in Ecology (B. C. Patten, ed.). p. 457. New York: Academic Press.

PLATT, J. (1969). In: Hierarchical Structures. (L. L. Whyte, A. G. Wilson \& D. Wilson, eds). p. 201. New York: Academic Press.

ROSEN, R. (1978). Fundamentals of Measurement and Representation of Natural Systems. New York: Elsevier North-Holland.

Shannon, C. E. \& Weaver, W. (1949). The Mathematical Theory of Communication. Urbana: University of Illinois Press.

SHAPIRO, J. (1973). Science 179, 382.

The Random House Dictionary of the ENGlish Language. (1968). New York: Random House.

WALLACE, R. L. (1978). Ecology 59, 221.

WIEGERT, R. G. \& OWEN, D. F. (1971). J. theor. Biol. 30, 69.

VON BERTALANFFY, L. (1962). Gen. Syst. 7, 1.

ZADEH, L. A. \& DESOER, C. A. (1963). Linear Systems The State Space Approach. New York: McGraw-Hill. 